\title{
Bleeding ileal varices: a rare cause of chronic anemia in liver cirrhosis
}

\author{
Elisabetta Castagna • Adele Cardellicchio • \\ Raffaella Pulitanò $\cdot$ Aldo Manca $\cdot$ Luigi Fenoglio
}

Received: 14 August 2010/Accepted: 14 September 2010/Published online: 8 October 2010

(C) SIMI 2010

\section{Case report}

A 70-year-old man was admitted to our hospital for severe microcytic anemia (Hb $5.9 \mathrm{~g} / \mathrm{dL}, \mathrm{MCV} 74 \mathrm{fl}$ ). The medical history included hepatic cirrhosis due to previous alcohol abuse with known portal hypertension, hemorrhoids and oesophageal varices. During the prior year, he had been admitted three times to the hospital due to ascites, treated with evacuative paracentesis for his microcytic anemia with low ferritinemia, each time he was transfused with blood and treated for 2 months with oral iron for an improvement in the hemoglobin level and in iron metabolism parameters. Two esophagogastroduodenoscopies (EGDs) and two colonoscopies did not show bleeding lesions, and even faecal occult blood testing was negative on multiple samples.

$\mathrm{He}$ was on treatment with canrenoate $400 \mathrm{mg} / \mathrm{die}$, furosemide $25 \mathrm{mg} / \mathrm{die}$, and propranolol $80 \mathrm{mg} / \mathrm{die}$. During immediate prior weeks, he reported general malaise, heart palpitations and weakness. He did not report any blood loss with stool, nor hematuria or hematemesis. He was afebrile, and the other vital signs were normal. He was pale, and there was no jaundice. Abdominal ultrasonography (US) showed slight free peritoneal fluid. $\mathrm{He}$ was initially

E. Castagna $(\bowtie)$

Scuola Specializzazione Medicina Interna, Università degli Studi di Torino, Torino, Italy

e-mail: elisc5@libero.it

A. Cardellicchio - L. Fenoglio

S.C. Medicina Interna, ASO S. Croce e Carle, via M. Coppino 26, Cuneo, Italy

R. Pulitanò · A. Manca

S.C. Gastroenterologia, ASO S. Croce e Carle,

via M. Coppino 26, Cuneo, Italy transfused with two units of packed red blood cells and an intravenous administration of iron was started. The faecal occult blood test on three samples was negative, a new EGDs showed oesophageal varices without signs of recent bleeding; colonoscopy revealed a normal mucosa with hemorrhoids.

In order to study the entire intestine, capsule endoscopy (CE) was performed; it revealed severe hyperemia of jejunoileal mucosa with multiple big blue varices in the ileum, without active bleeding (Fig. 1).

Due to the persistence of anemia, and the need for frequent blood transfusion, along with a lack of response to betablockers, a transjugular intrahepatic portosystemic shunt (TIPSS) procedure was performed with no complications. The patient underwent a CE 5 days after showing the disappearance of the multiple varices (Fig. 2); the anemia improved quickly, and no further blood transfusion has been necessary up to the present.

\section{Discussion}

Portal hypertensive enteropathy (PHE) is present in 5-11\% of patients with portal hypertension and chronic active bleeding [1]; the presence of varices and of areas of mucosa with a reticulate pattern should be considered manifestations of portal hypertension in the small bowel. Ectopic varices are an unusual cause of gastrointestinal hemorrage and can account for up to $5 \%$ of variceal bleeding; acute bleeding is associated with a high mortality. These varicescan occur at different sites in the gastrointestinal tract, including the duodenum, small bowel, colon and peristomal locations.

Endoscopic diagnosis of ileal PHE and ileal varices is impossible to achieve with a standard endoscope, and 


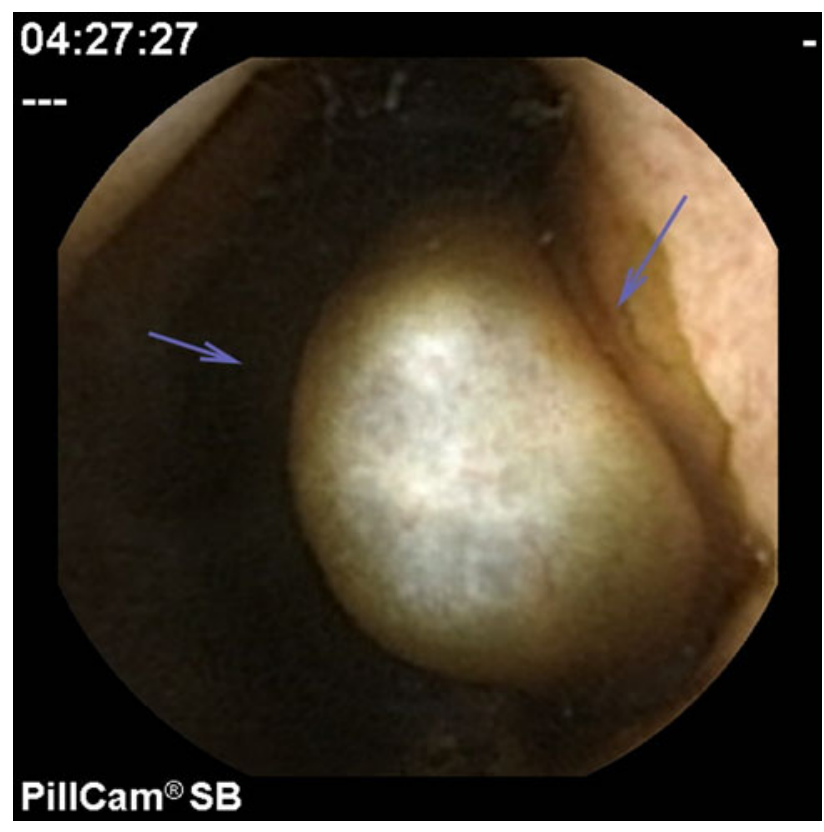

Fig. 1 Capsule endoscopy showing ileal varices

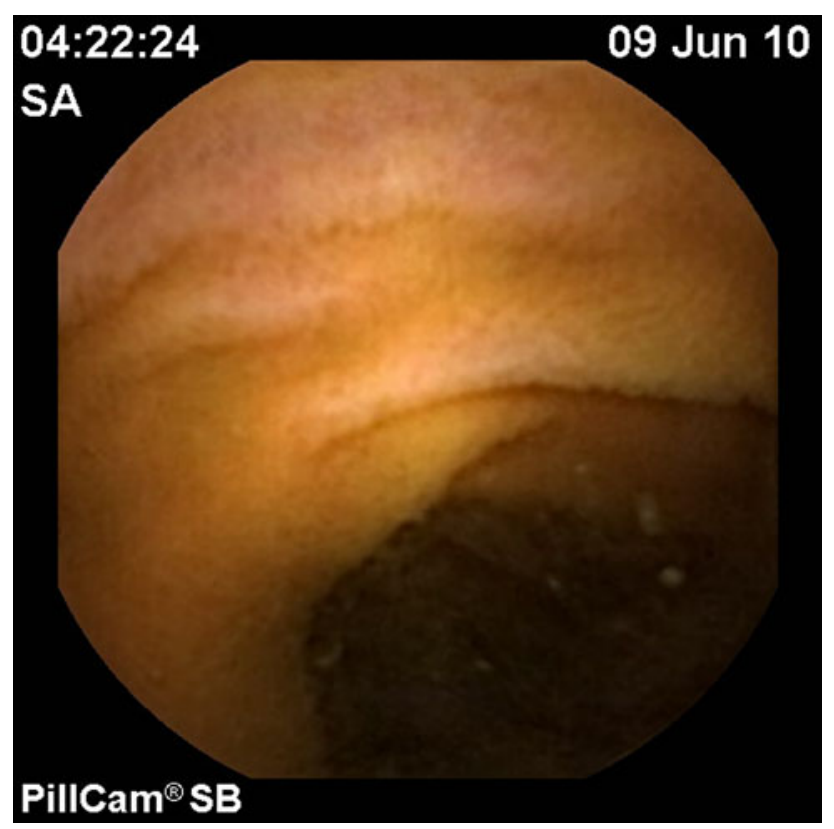

Fig. 2 Normal ileal mucosa after TIPSS

mesenteric angiography often gives negative results. With the advent of capsule endoscopy (CE), it is now possible to examine the entire small intestine without discomfort to the patient and on an outpatient ambulatory basis. This method does not, however, permit biopsies nor treatment, a limitation that has been overcome by double and single-balloon enteroscopy [2].

The bleeding from these ectopic sources can be hard to manage due to an initial difficulty in determining the source of the bleeding, as well as the subsequent difficulty in application of therapy due to inaccessibility of the varices in some locations. The available evidence for the management of bleeding ectopic varices is limited to case reports or small series, with no randomized-controlled trials. The options include medical therapies such as the use of vasopressin analogues or octreotide [1], and endoscopic therapies such as sclerotherapy, thrombin injection, and injection of tissue adhesives or ligation. Other treatments utilized include embolization using steel coils, and resection of the bowel segment containing the varix. Balloonoccluded retrograde transvenous obliteration has also been tried.

All these procedures have limited success in achieving initial haemostasis, and re-bleeding rates following these procedures are high. With all these treatments, the underlying portal hypertension remains uncorrected, and thus the patients remain at risk for re-bleeding from ectopic varices due to continued formation of new feeding collateral vessels. To treat the underlying portal hypertension, the performance of a TIPSS is recommended [3]. TIPSS is now the first line treatment for refractory ectopic variceal bleeding [4].

Rates of re-bleeding from ectopic varices in literature are $21-23 \%$ at 1 year post TIPSS. [5]. None of the factors such as Child-Pugh score, pre-TIPSS or the type of stent used (covered-uncovered) are significantly associated with either risk of recurrence of bleeding from ectopic varices or death in the larger series.

Re-bleeding can be related to shunt dysfunction, and responds to shunt interventions. Therefore, the shunt should be investigated first in case of re-bleeding. Nevertheless, patients with ectopic varices may re-bleed after TIPSS despite initial achievement of hemodynamic targets and the presence of a patent functioning shunt in situ. In some series, re-bleeding more commonly occurs in those patients who have a TIPSS procedure without concomitant variceal embolization (VE) [4] versus those who do, but this is still controversial as other series do not report this [5]. In the event of re-bleeding, if the varices are accessible, local treatment may be tried first. If these measures are ineffective or not possible and if the varices are accessible radiologically, VE may be tried. The procedure-related complications rate is generally low (about 3\%) [5].

Acknowledgments The authors would like to acknowledge the entire staff of the Service of Endoscopy of the S. Croce Hospital in Cuneo.

Conflict of interest None.

\section{References}

1. De Palma GD, Rega M, Masone S et al (2005) Mucosal abnormalities of the small bowel in patients with cirrhosis and 
portal hypertension: a capsule endoscopy study. Gastrointest Endosc 62:529-534

2. Traina M, Tarantino I, Mocciaro F (2009) Variceal bleeding from ileum identified and treated by single balloon enteroscopy. World $\mathrm{J}$ Gastroenterol 15:1904-1905

3. Kochar N, Tripathi D, Mcavoy NC et al (2008) Bleeding ectopic varices in cirrhosis: the role of transjugular intrahepatic portosystemic stent shunts. Aliment Pharmacol Ther 28:294-303
4. Vangeli M, Patch D, Terreni N et al (2004) Bleeding ectopic varices-treatment with tranjugular intrahepatic porto-systemic shunt (TIPS) and embolisation. J Hepatol 41:560-566

5. Vidal V, Joly L, Perreault P et al (2006) Usefulness of tranjugular intrahepatic portosystemic shunt in the management of bleeding ectopic varices in cirrhotic patients. Cardiovasc Intervent Radiol 29:216-219 